\title{
Glyphosat geprägte Polymere als kostengünstige und robuste künstliche selektive Sorptionsmittel für die Modifizierung von low-cost Sensoren
}

\author{
Mashaalah Zarejousheghani ${ }^{1,2}$, Parvaneh Rahimi ${ }^{1}$, Helko Borsdorf ${ }^{2}$, Stefan Zimmermann ${ }^{3}$ and Yvonne \\ Joseph $^{1}$ \\ ${ }^{1}$ Instituts für Elektronik- und Sensormaterialien, Fakultät für Werkstoffwissenschaft und Werkstofftechnologie, \\ Technische Universität Bergakademie Freiberg, Freiberg, Deutschland \\ ${ }^{2}$ Department Monitoring and Exploration Technologies, Helmholtz Centre for Environmental Research-UFZ, \\ Leipzig, Deutschland \\ ${ }^{3}$ Department of Sensors and Measurement Technology, Institute of Electrical Engineering and Measurement \\ Technology, Leibniz University Hannover, Hannover, Deutschland \\ mashaalah.zarejousheghani@esm.tu-freiberg.de
}

\section{Einleitung}

Weltweit wächst die Besorgnis über Gesundheitsrisiken, die von der Kontamination der natürlichen Wasserressourcen ausgehen. Die dauerhafte Exposition gegenüber bestimmten Chemikalien kann schwere und dauerhafte gesundheitliche Folgen haben, die sich durch eine frühzeitige Erkennung leicht vermeiden lassen. So steht Glyphosat, das weltweit am häufigsten verwendeten Herbizid, im Verdacht, krebserregend zu sein und wird zudem maßgeblich für das Insektensterben verantwortlich gemacht. Der Nachweis von Glyphosat ist aufgrund seiner hohen Polarität in wässrigen Lösungen und des Fehlens von Absorptions- oder Fluoreszenzeigenschaften sehr schwierig. Daher ist eine verbesserte Glyphosat-Analyse erforderlich [1]. Von den selektiven künstlichen Bio(mimetik)rezeptoren gehören molekular geprägte Polymere (MIPs) zu den vielversprechenden Kandidaten für die Modifizierung von Sensoren, insbesondere von Einwegsensoren, da sie kostengünstig und einfach herzustellen, leicht zu konstruieren und langzeitstabil sind. Sie eignen sich für eine Vielzahl von Zielananlyten [2].

MIPs können mit kovalenten, nicht-kovalenten oder semikovalenten Methoden synthetisiert werden [3]. Aufgrund der inhärenten Vorteile des nicht-kovalenten Ansatzes ist dies immer noch die an der häufigsten verwendeten Methode zur Entwicklung geprägter Polymere. Beim nichtkovalenten Ansatz sind die meisten intermolekularen Wechselwirkungen, die für die Anordnung der funktionellen Monomere um die Templatmoleküle verantwortlich sind, Wasserstoffbrücken, elektrostatische oder ionische Wechselwirkungen, Van-der-Waals-Kräfte (VDW), т-т-Wechselwirkungen und hydrophobe Effekte. Während die Wasserstoffbrücken-Wechselwirkung die am häufigsten genutzte nicht-kovalente intermolekulare Wechselwirkung ist, können Wechselwirkungen zwischen entgegengesetzt geladenen lonen zu stärkeren intermolekularen Wechselwirkungen führen (kovalente Bindungen: $\sim 500 \mathrm{~kJ} / \mathrm{mol}$; ionische Wechselwirkung: bis zu $60 \mathrm{~kJ} / \mathrm{mol}$; Wasserstoffbrückenbindung: bis zu $40 \mathrm{~kJ} / \mathrm{mol}$, Ladungs-Dipol-Wechselwirkungen: bis zu $8 \mathrm{~kJ} / \mathrm{mol}$; Dipol-Dipol-Wechselwirkung: $\sim 1 \mathrm{~kJ} / \mathrm{mol}$; VDW-Wechselwirkungen: 0,1-1 kJ/mol) [4]. Wir haben bereits die Anwendbarkeit der ionischen Wechselwirkung für die Synthese eines hochselektiven geprägten Polymers für negativ geladenes Acesulfam, einen bekannten anthropogenen Marker, unter Verwendung von (Vinylbenzyl)trimethylammoniumchlorid (VBTA) als effizientes lonenpaar-Reagenz gezeigt [5-7]. Wir glauben, dass quaternäre Ammoniumkationen mit Vinylgruppen zu den effizientesten und praktischsten funktionellen Monomeren gehören, die die Synthese selektiver geprägter Polymere für negativ geladene Zielmoleküle wie Glyphosat ermöglichen könnten.

Zur Prägung glyphosatempfindlicher Polymere als wichtige Modellverbindung für elektrisch geladene und gut wasserlösliche chemische Verbindungen wurde ein kleiner Pool von quaternären Ammoniumkationen enthaltenden funktionellen Monomeren systematisch genutzt. Die gleichzeitigen Wechselwirkungen zwischen drei positiv geladenen Monomeren und Glyphosat wurden dabei zunächst mit Hilfe einer statistischen Experimentplanungsmethode bewertet. Anschließend wurden die verschiedenen geprägten Polymere an der Goldoberfläche einer Quarzkristall-Mikrowaage (QCM)mit optimierten und nicht optimierten Glyphosat-Monomer-Verhältnissen synthetisiert. Die funktionellen Monomere erwiesen sich als hocheffizient, wenn sie zusammen und im optimierten Verhältnis verwendet werden. Die modifizierte QCM wurde für die Bestimmung von Glyphosat in Wasserproben verwendet und mit Standardanalysenmethoden verglichen. QCM mit synthetisiertem MIP zeigte eine deutliche Verbesserung der Selektivität und Empfindlichkeit des Sensors gegenüber Glyphosat im Vergleich zu (Aminomethyl)phosphonsäure (AMPA), die als primäres Abbauprodukt von Glyphosat bekannt ist [8].

\section{Methoden und Materialien}

Die in dieser Studie verwendeten Chemikalien waren Ethylenglykoldimethacrylat (EGDMA) (CAS-Nr. 97-90-5), 2,2'-Azobis(2-methylpropionitril) (AIBN) (CAS-Nr. 78-67-1), (3-Acrylamidopropyl)trimethylammoniumchlorid-Lösung (75 Gew.-\% in H2O) (CAS-Nr. 45021-77-0), [2-(Acryloyloxy)ethyl]trimethylammoniumchlorid-Lösung (80 Gew.-\% in $\mathrm{H} 2 \mathrm{O}$ ) (CAS-Nr. 44992-01-0), Diallyldimethylammoniumchlorid (CAS-Nr. 7398-69-8), Glyphosat (CAS-Nr. 1071-836), (Aminomethyl)phosphonsäure (AMPA) (CAS-Nr. 106651-9), Dichlordimethylsilan (DCDMS) (CAS-Nr. 75-78-5), 9Fluorenylmethoxycarbonylchlorid (Fmoc-Cl 97\%) (CAS-Nr. 28920-43-6) und Borax wasserfrei (CAS-Nr. 1330-43-4) 
wurden von Sigma-Aldrich bezogen. Chloroform (CAS-Nr. 67-66-3), Acetonitril (CAS-Nr. 75-05-8), Schwefelsäure 95 $\%$ (CAS-Nr. 7664-93-9) und Wasserstoffperoxidlösung 30 \% (CAS-Nr. 7722-84-1) wurden von MERCK (Darmstadt, Deutschland) bereitgestellt. Allylmercaptan (>70,0 \%) (CAS-Nr. 870-23-5) wurde von Tokyo Chemical Industry Co, Ltd. (TCl) beschafft.

Die folgenden Instrumente wurden für die Synthese und Bewertung der geprägten und nicht geprägten Polymere verwendet: eine VL-6LMUV-Lampe ( $6 \mathrm{~W}, 312 \mathrm{~nm}$ ), ein Topometrix TMX-2010 Rasterkraftmikroskop (AFM), ein TENSOR II von Bruker (Leipzig, Deutschland), ein Tropfenkonturanalysator von KRÜSS GmbH (Hamburg, Deutschland), ein Niederdruckplasmasystem von Diener electronic $\mathrm{GmbH}$ (Ebhausen, Deutschland), eine Röntgenphotoelektronenspektroskopie, die auf einem Thermo Fisher Scientific ESCALAB 250Xi Spektrometer durchgeführt wurde, die goldbeschichteten $5 \mathrm{MHz}$ QCM-Sensorkristalle von MicroVacuum (Budapest, Ungarn) und eine kommerzielle mikrofluidische Quarzbeschichtungszelle von QSense mit laminarer Strömung und einem Volumen von $140 \mu \mathrm{L}(40 \mu \mathrm{L}$ über dem Quarz) wurde für die Fließinjektionsanalyse (FIA) verwendet.

Central Composite Design (CCD) wurde zur statistischen verwendet, um die gesamten Parameter der Prozesse mit einer begrenzten Anzahl von Versuchen zu untersuchen. In dieser Untersuchung wurden die Freiheitsgrade durch 16 Durchläufe abgebildet, wobei jeder Durchlauf eine andere Kombination der Mengen an quaternären Ammoniumkationen bei konstanter Glyphosatkonzentration aufweist.

Tab. 1: Glyphosat und funktionelle Monomere

\begin{tabular}{|c|c|c|}
\hline Glyphosate & & \\
\hline $\begin{array}{l}\text { (3-Acrylamidopropyl)trime- } \\
\text { thylammonium chloride }\end{array}$ & 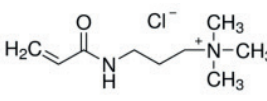 & A \\
\hline $\begin{array}{l}\text { [2-(Acryloyloxy)ethyl]tri- } \\
\text { methylammonium chloride }\end{array}$ & 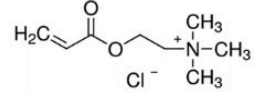 & B \\
\hline $\begin{array}{l}\text { Diallyldimethylammo- } \\
\text { nium chloride }\end{array}$ & $\begin{array}{lll}\mathrm{CH}_{+}^{+} & \mathrm{Cl} \\
\mathrm{CH}_{3} & & \\
\mathrm{CH}_{3} & & \\
\end{array}$ & $\mathrm{C}$ \\
\hline
\end{tabular}

Glyphosat und funktionelle Monomere wurden gemischt und die optimalen Verhältnisse wurden mit Hilfe der CCD ermittelt. Um die optimierten und nicht optimierten Verhältnisse zu vergleichen, wurden beide verwendet, um molekular geprägte und nicht geprägte Polymere auf der Goldoberfläche von QCMs zu synthetisieren.

\section{Ergebnisse}

Abbildung 1 zeigt Kontaktwinkelmessungen der nicht modifizierten $(\mathrm{Au})$ und modifizierten QCMs (AM: Allylmercaptan, MIP: Molecularly imprinted polymer, NIP: non imprinted polymer; A, B, C: verwendete Monomere).

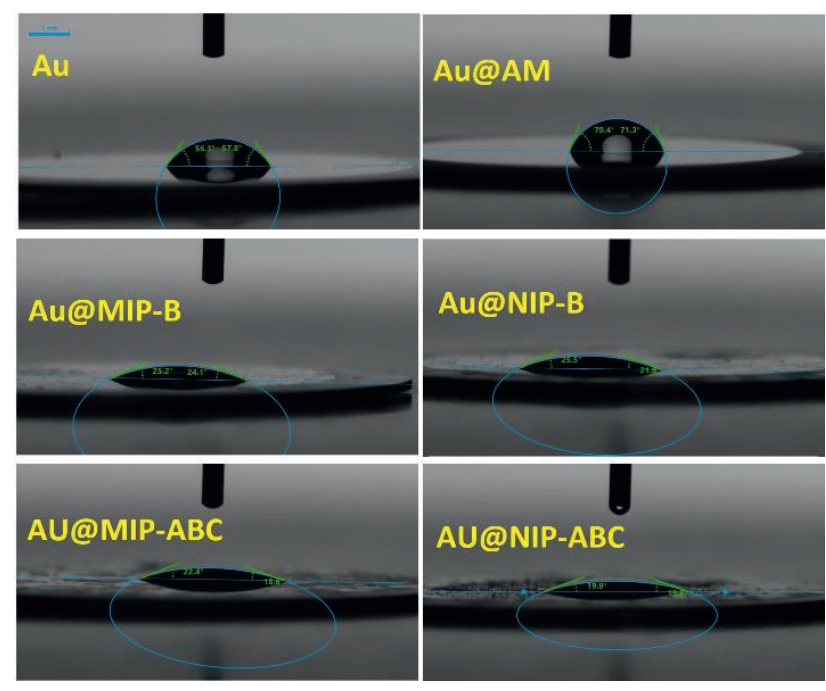

Abb. 1: Kontaktwinkel-Bilder für Au- und QCMs

Die Abbildung 2 zeigt die FTIR Spektren für die nicht modifizierte und die modifizierten QCM.
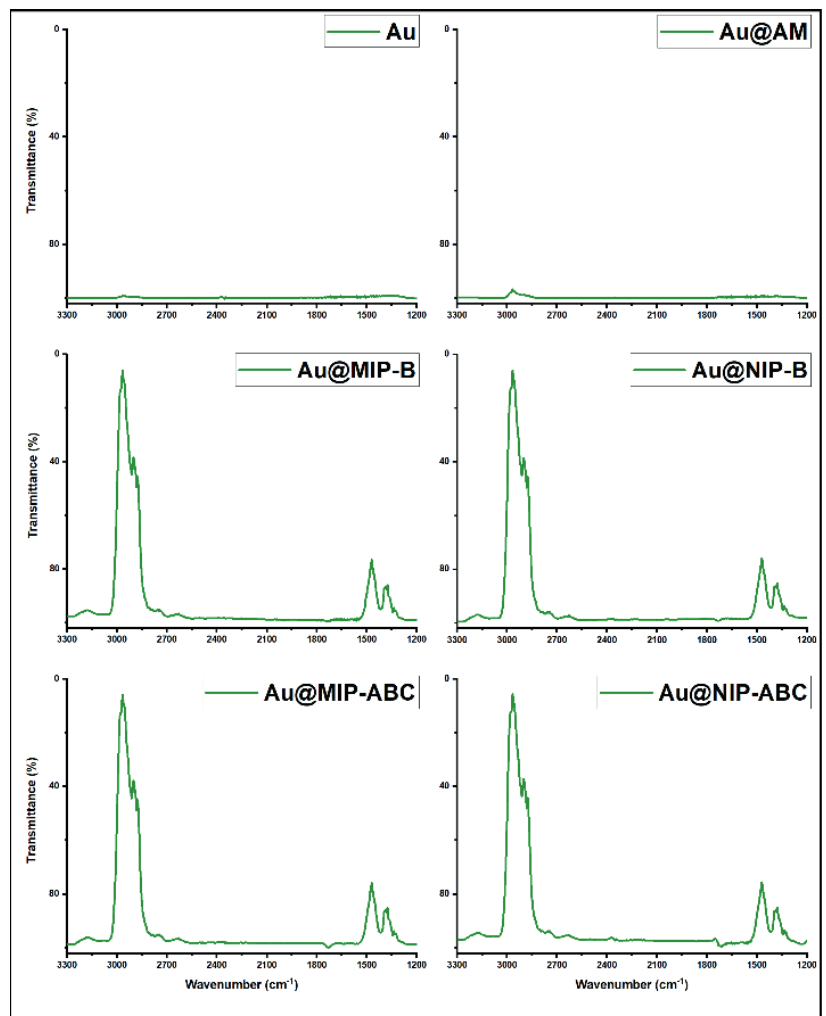

Abb. 2: FTIR Spektren für Au- und QCMs 

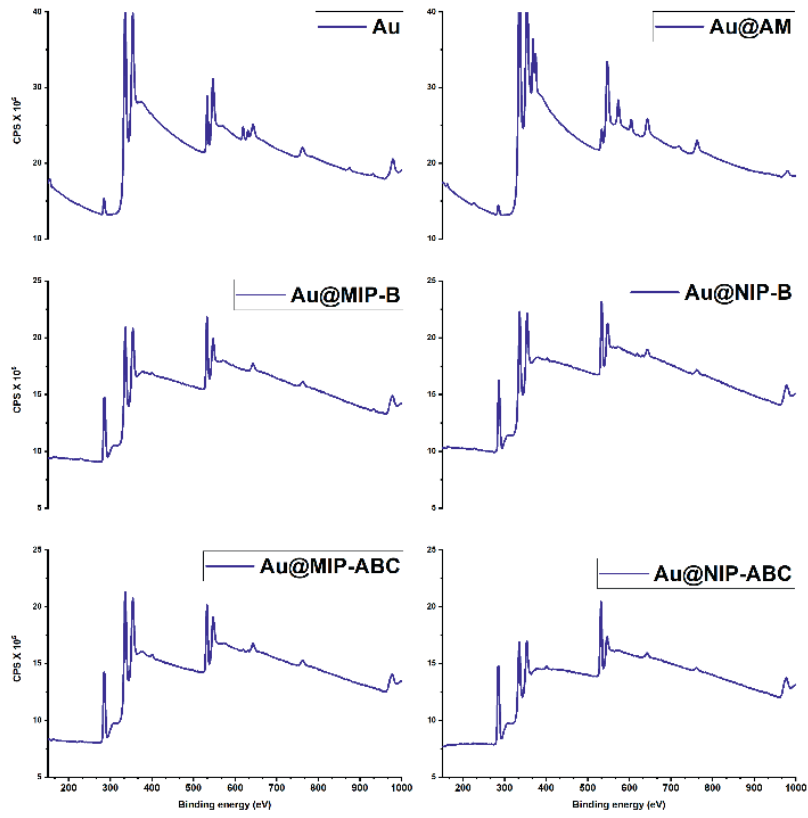

Abb. 3: XPS Spektren für Au- und QCMs

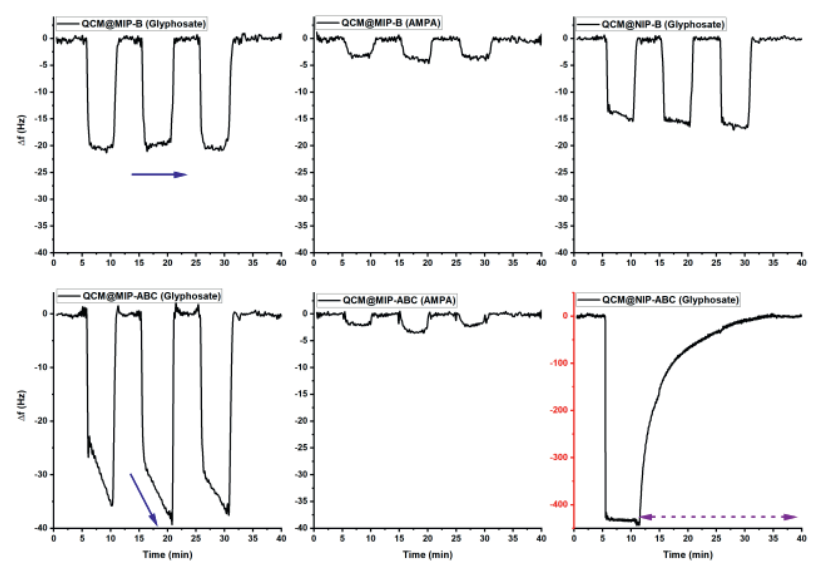

Abb. 4: Messungen von Glyphosat und AMPA bei $3 \mathrm{mM}$ mit QCMs, die mit verschiedenen geprägten und nicht geprägten Polymeren modifiziert wurden

Die Abbildung 3 zeigt die XPS Spektren für die nicht modifizierte und die modifizierten QCM und Abbildung 4 zeigt die QCM Messungen für Glyphosat und AMPA.

\section{Diskussion}

Glyphosat ist eine polare Chemikalie, die in Wasser sehr gut löslich ist, insbesondere bei höheren $\mathrm{pH}$-Werten. Unter alkalischen Bedingungen kann das Glyphosatmolekül leicht deprotoniert werden und ein Molekül bilden, das mit negativen lonen elektrisch geladen ist. Sowohl Glyphosat als auch Kationen (A, B und C) wurden in alkalischem Wasser aufgelöst und ihre Wechselwirkungen systematisch mit der statistischen CCD-Methode bewertet. Die Ergebnisse zeigten, dass das Kation B die effizienteste Wechselwirkung mit Glyphosat aufweist. Das Kation A könnte ebenfalls mit Glyphosat interagieren, jedoch in geringerer Stärke. Bei höherer Konzentration könnte Kation A sogar negative Auswirkungen haben. Das optimale Kationenverhältnis für $1 \mathrm{mmol}$ Glyphosat wurde mit $B=3 \mathrm{mmol}, C=3 \mathrm{mmol}$ und $A=2$ mmol ermittelt.

Nach Bewertung der Wechselwirkungen zwischen Glyphosat und Kationen wurden zwei verschiedene geprägte Polymere und ihre verwandten nicht geprägten Polymere synthetisiert, wobei i) das Verhältnis der Kationen Glyphosat:B:A:C (1:3:3:2 mmol) und ii) Glyphosat:B (1:3 mmol) optimiert wurden.

Abbildung 1 zeigt die Veränderungen der Kontaktwinkel von QCMs vor und nach der Modifikation. Die Oberfläche der QCM wurde mit FTIR- und XPS-Methode charakterisiert. Die Ergebnisse zeigten, dass FTIR nicht empfindlich genug war und die XPS-Ergebnisse belegten die Oberflächenmodifikationen mit verschiedenen geprägten und nicht geprägten Polymeren (Abbildungen 2-3).

Die Bindung von Glyphosat und seinem primären Abbauprodukt, AMPA, wurde an der Oberfläche von geprägten und nicht geprägten Polymeren untersucht (Abbildung 4). Die Ergebnisse zeigen, dass das geprägte Polymer, das nur mit dem Kation B (MIP-B) synthetisiert wurde, eine Selektivität gegenüber Glyphosat aufweist. Es kann die Zielsubstanz sehr schnell adsorbieren und die adsorbierte Zielsubstanz kann auch leicht abgewaschen werden. Die mit MIP-B und NIP-B modifizierten QCMs wurden zur Messung von Glyphosat in verschiedenen Konzentrationen verwendet.

Bei MIP-ABC ist während der Probeninjektion eine starke Diffusion zu beobachten, was zeigt, dass die Glyphosatmoleküle stark von der MIP-ABC-Polymermatrix angezogen werden. Andererseits konnte das nicht geprägte Polymer, das mit drei Kationen synthetisiert wurde (NIP-ABC), Glyphosatmoleküle deutlich besser adsorbieren, als das entsprechend geprägte Polymer. Interessanterweise benötigt das an NIP-ABC adsorbierte Glyphosat (während 5 Minuten) eine längere Zeit zur vollständigen Desorption $(\approx 35$ Minuten). Anhand dieser interessanten Daten haben wir ein einfaches Modell entwickelt, das die Wechselwirkungen beschreibt, die die Glyphosatadsorption durch verschiedene synthetische Polymere steuern (Abbildung 5). Wir glauben, dass auf der Grundlage dieses Modells empfindlichere und selektivere modifizierte Sensoren entwickelt werden könnten. 

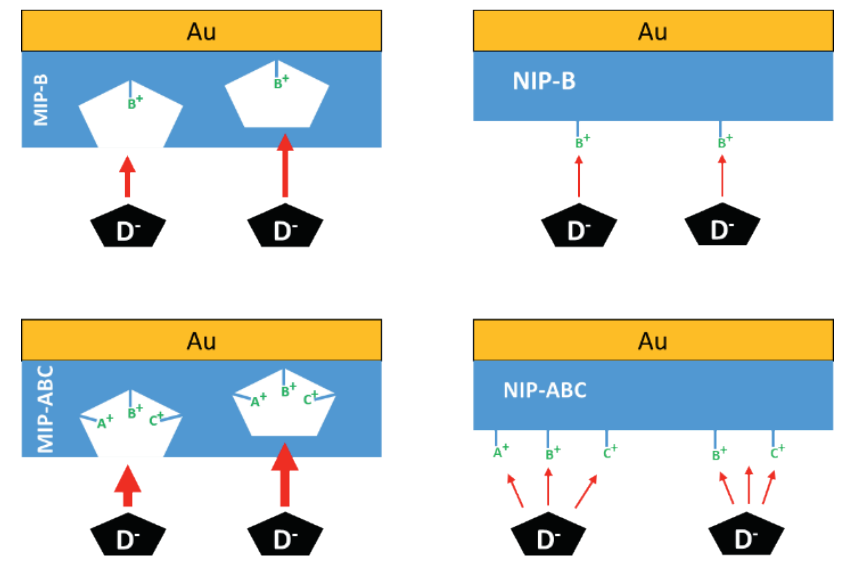

Abb. 5: Ein einfaches Modell, das die Wechselwirkungen beschreibt, die die Adsorption von Glyphosat durch verschiedene synthetische Polymere steuern.

Um einen empfindlichen Sensor für die schnelle Überwachung von Glyphosatmolekülen zu entwickeln, muss die Dichte der Erkennungsstellen auf der Oberfläche erhöht werden. Zu diesem Zweck könnten die mit der QCM mit NIP-ABC erzielten Ergebnisse als Inspiration für neue Ideen dienen. Glyphosatmoleküle konnten stark an der Oberfläche dieses Polymers adsorbieren. Dies deutet darauf hin, dass Glyphosatmoleküle als Chelatbildner stark mit zufällig verteilten und stabilisierten Kationen an der Oberfläche des NIP-ABC-Polymers interagieren können. Angeregt durch diese interessanten Ergebnisse wollen wir eine neue Synthesestrategie für die Herstellung hochselektiver geprägter Polymere für gut wasserlösliche Verbindungen entwickeln. Diese neue Strategie ist die 2-D-Polymerisation an der Oberfläche der Sensoren.

\section{Literatur}

[1] Huhn, C. More and enhanced glyphosate analysis is needed. Anal. Bioanal. Chem. 2018, 410, 3041-3045, doi:10.1007/s00216-018-1000-3.

[2] Zarejousheghani, M.; Rahimi, P.; Borsdorf, H.; Zimmermann, S.; Joseph, Y. Molecularly Imprinted PolymerBased Sensors for Priority Pollutants. Sensors 2021, 21, 2406, doi:10.3390/s21072406

[3] Zarejousheghani, M.; Lorenz, W.; Vanninen, P.; Alizadeh, T.; Cämmerer, M.; Borsdorf, H. Molecularly Imprinted Polymer Materials as Selective Recognition Sorbents for Explosives: A Review. Polymers (Basel) 2019, 11, doi:10.3390/polym11050888.

[4] MOLECULARLY IMPRINTED MATERIALS. Science and technology; CRC PRESS: [S.I.], 2020, ISBN 0367578190

[5] Zarejousheghani, M.; Schrader, S.; Möder, M.; Mayer, T.; Borsdorf, H. Negative electrospray ionization ion mobility spectrometry combined with paper-based molecular imprinted polymer disks: A novel approach for rapid target screening of trace organic compounds in water samples. Talanta 2018, 190, 47-54, doi:10.1016/j.talanta.2018.07.076.
[6] Zarejousheghani, M.; Schrader, S.; Möder, M.; Schmidt, M.; Borsdorf, H. A new strategy for accelerated extraction of target compounds using molecularly imprinted polymer particles embedded in a paper-based disk. J. Mol. Recognit. 2018, 31, doi:10.1002/jmr.2629.

[7] Zarejousheghani, M.; Schrader, S.; Möder, M.; Lorenz, P.; Borsdorf, $H$. lon-exchange molecularly imprinted polymer for the extraction of negatively charged acesulfame from wastewater samples. J. Chromatogr. A 2015, 1411, 23-33, doi:10.1016/j.chroma.2015.07.107.

[8] Zarejousheghani, M.; Jaafar, A.;Wollmerstaedt, H.; Rahimi, P.; Borsdorf, H.; Zimmermann, S.; Joseph, Y. Rational Design of Molecularly Imprinted Polymers Using Quaternary Ammonium Cations for Glyphosate Detection. Sensors 2021, 21, 296, doi: $10.3390 / \mathrm{s} 21010296$ 